\title{
Parâmetros Nutricionais entre Homens e Mulheres Idosos com Doença Renal Crônica em Tratamento Hemodialítico
}

\author{
Emili Paixão Trenhago, ${ }^{1}$ Luana Fioravanti Roland, ${ }^{2}$ \\ Mariane Rosa, ${ }^{2}$ Loiva Beatriz Dallepiane ${ }^{3}$
}

\begin{abstract}
RESUMO
Pacientes idosos em hemodiálise estão sujeitos às modificações alimentares, antropométricos e bioquímicas que são provocadas pela própria doença renal, pelo tratamento hemodialítico e pelo processo de envelhecimento. Neste trabalho objetivou-se avaliar a relação dos parâmetros nutricionais entre homens e mulheres idosos em tratamento hemodialítico. Em estudo transversal realizado em Santa Maria (RS), foram considerados elegíveis para a pesquisa idosos com idade a partir de 60 anos, de ambos os sexos, diagnosticados com doença renal crônica, que realizavam hemodiálise três vezes na semana e em tratamento há pelo menos três meses. Como parâmetros nutricionais, foram considerados os dados antropométricos, dietéticos e exames bioquímicos. Para a comparação entre os sexos masculino e feminino, das variáveis paramétricas foi utilizado o Teste $t$ de Student bicaudal para amostras independentes. Os resultados encontrados indicam relação do sexo com o índice de massa corporal, quilocalorias, proteína, carboidrato, fósforo, potássio, vitamina D e Kt/V. A partir disso, pode-se concluir que os achados deste estudo reforçam a importância da avaliação nutricional no contexto do tratamento de renais crônicos em hemodiálise, visando a uma boa adesão à dieta e adequação à diálise.
\end{abstract}

Palavras-chave: Insuficiência renal crônica. Diálise renal. Nutrição do idoso.

\section{NUTRITIONAL PARAMETERS IN BETWEEN OLD MEN AND WOMEN WITH CHRONIC KIDNEY DISEASE IN HEMODIALITIC TREATMENT}

\section{ABSTRACT}

Hemodialysis patients elderly are subject to dietary changes anthropometric and biochemical that are caused by their own kidney disease, hemodialysis treatment and the aging process. The objective of this study was to evaluate the relationship of nutritional parameters between elderly men and women undergoing hemodialysis. In a cross-sectional study conducted in Santa Maria-RS, were considered eligible for the search elderly over 60 years of both sexes, diagnosed with chronic kidney disease who underwent hemodialysis three times a week and in treatment for at least three months. As nutritional parameters were considered anthropometric, dietary data and biochemical tests. For the comparison between male and female, the parametric variables we used the Test $t$ de Student bicaudal for independent samples. The results indicate relationship between body mass index and sex, kilocalories, protein, carbohydrate, phosphorus, potassium, sodium and vitamin D. It was possible to conclude that the findings of this study reinforce the importance of nutritional assessment in the context of the treatment of chronic kidney in hemodialysis, aiming at a good adherence to the diet and adaptation to dialysis.

Keywords: Renal insufficiency chronic. Renal dialysis. Elderly Nutrition.

\footnotetext{
${ }^{1}$ Universidade Federal de Santa Maria (UFSM) - Campus Palmeira das Missões. Palmeira das Missões/RS, Brasil.

${ }^{2}$ Universidade Federal de Santa Maria (UFSM). Santa Maria/RS, Brasil.

${ }^{3}$ Autora correspondente. Universidade Federal de Santa Maria (UFSM) - Campus Palmeira das Missões. Av. Independência, 3751 - Vista Alegre, $98300-000$. Palmeira das Missões/RS, Brasil. http://lattes.cnpq.br/5570695730349223. https://orcid.org/0000-0003-1331-2882. loiva.dallepiane@hotmail.com
} 


\section{INTRODUÇÃO}

A transição demográfica, decorrente da redução das taxas de mortalidade e das taxas de natalidade, ocasionou modificações na estrutura etária da população (MIRANDA; MENDES; SILVA, 2016). A população mundial era de 7,6 bilhões em $2017,61 \%$ correspondente à idade entre 15 e 59 anos e $13 \%$ correspondendo aos idosos com mais de 60 anos, ou seja, a expectativa de vida dos idosos teve ganhos significativos nos últimos anos, com aumento de 3,6 anos entre $2000-2005$ e $2010-2015$, de 67,2 para 70,8 anos (NATIONAL..., 2019).

O envelhecimento é considerado um processo mundial, dinâmico e irreversível, instigado por fatores biológicos, sociais, psicológicos e ambientais, e apresenta implicações como o declínio na força dos músculos esqueléticos, acometendo, principalmente, a capacidade funcional do indivíduo (BORGES et al., 2015).

$O$ processo de envelhecimento pode estar relacionado com Doenças Crônicas não Transmissíveis (DCNT), que favorecem o desenvolvimento de incapacidades motoras, psicológicas e sociais, impedindo os idosos de realizarem suas atividades cotidianas, e são responsáveis por cerca de $70 \%$ da mortalidade no Brasil (BARRETO; CARREIRA; MARCON, 2015; MENDES et al., 2018). Dentre elas, destaca-se a Doença Renal Crônica (DRC), considerada um problema de saúde pública com relevante impacto econômico. Essa condição, em 1990, respondia pela 17a causa de morte no Brasil e, em 2010, já ocupava a 10a colocação. Além disso, ocorreu um aumento significativo na contribuição da doença renal crônica como causa de morte prematura em mulheres (MARINHO; PASSOS; FRANÇA, 2016).

A hipertensão e o diabetes estão dentre as causas da DRC que ocasiona falência dos rins e necessidade de terapia renal substitutiva, acarretando uma enorme obrigação ao sistema de saúde. Dentre os tipos de terapia substitutiva, a hemodiálise (HD) corresponde à principal escolha, sendo empregada em 91\% dos casos (SESSO et al., 2016).

Segundo o Inquérito Brasileiro de Diálise Crônica, em 2017 foi estimado um quantitativo de aproximadamente 126.583 pacientes em diálise crônica no Brasil. O relatório apresentou um aumento constante do número de pacientes em diálise, relacionado com o Inquérito Brasileiro de Diálise Crônica em 2016, cujo número estimado foi de 122.825 pacientes em diálise, e o percentual de idosos ( $\geq 65$ anos) em diálise crônica em 2016 atingiu 33\% do total (THOMÉ et al., 2019; SESSO et al., 2016).
Os idosos em tratamento hemodialítico estão sujeitos a deficiências nutricionais, principalmente quando submetidos a situações de estresse ou doença. A desnutrição proteico-calórica é uma das disfunções mais prevalecentes nos pacientes em tratamento hemodialítico, estando relacionada com o aumento da morbidade e da mortalidade. Sua origem é multifatorial e engloba aspectos ligados ao consumo alimentar insuficiente, acidose metabólica, distúrbios hormonais e gastrointestinais, medicamentos que influenciam na absorção de alimentos, doenças intercorrentes e perda de nutrientes durante o tratamento dialítico (DUARTE, 2017). Nesse contexto, a problematização do estudo consiste em saber se há diferença nos parâmetros nutricionais entre homens e mulheres idosos em tratamento hemodialítico, posto que para o melhor acompanhamento dietético é necessário uma adesão às modificações na dieta e, por isso, justifica-se o interesse para saber se há diferenças considerando idosos do sexo masculino e feminino, e, também, porque no Inquérito Brasileiro de Diálise Crônica 2017 (THOMÉ et al., 2019) a prevalência de homens em hemodiálise é de $58 \%$, ou seja, maior que as mulheres.

Assim, considerando o envelhecimento populacional, o aumento do número de idosos em hemodiálise e a vulnerabilidade destes ao equilíbrio nutricional, este estudo tem como objetivo avaliar a relação dos parâmetros nutricionais entre homens e mulheres idosos em tratamento hemodialítico.

\section{METODOLOGIA}

Trata-se de um estudo transversal com abordagem descritiva e analítica, resultante do projeto "Indicadores nutricionais e qualidade de vida de idosos com doença renal crônica em tratamento hemodialítico", aprovado pelo Comitê de Ética em Pesquisa da Universidade Federal de Santa Maria (UFSM) sob o ${ }^{\circ}$ 2.545.211, realizado no período de março a julho de 2018 na cidade de Santa Maria (RS) em duas unidades de uma clínica renal, que é referência no atendimento de pacientes procedentes da região central do Rio Grande do Sul.

Foram considerados elegíveis para o estudo idosos com idade a partir de 60 anos, de ambos os sexos, diagnosticados com DRC, que realizavam HD três vezes na semana, em tratamento há pelo menos três meses e que não apresentaram dificuldade de compreensão na entrevista, avaliados pelo teste de evocação de três palavras. Foram excluídos da pesquisa os pacientes que utilizavam nutrição enteral. 
As variáveis analisadas foram os dados sociodemográficos para caracterização da amostra e parâmetros nutricionais. Como parâmetros nutricionais foram considerados os dados antropométricos, dietéticos e exames bioquímicos. As medidas antropométricas utilizadas foram o peso corporal seco e a estatura a partir do prontuário do paciente para posterior cálculo do índice de massa corporal (IMC), classificadas de acordo com os parâmetros da Organização Pan-Americana de Saúde (OPAS, 2001).

Foram aferidas também as medidas da circunferência do braço $(C B)$ e circunferência da panturrilha (CP) e classificadas segundo a Organização Mundial de Saúde (WHO..., 1995). Os dados dietéticos foram avaliados por meio de três recordatórios de 24 horas em dias alternados, sendo um dia em que o paciente se submeteu à sessão de hemodiálise e dois dias sem o procedimento, incluindo um domingo. Adotou-se esta técnica em razão de que um único recordatório não estima a dieta habitual, e a ingestão relatada pode ser atípica em apenas um dia.

Para a avaliação energética de macronutrientes (proteínas, carboidratos e lipídeos) e micronutrientes (fósforo, potássio, cálcio, ferro e sódio) foi utilizada a média dos três dias calculada no software CalcNut (DA COSTA, 2009). A adequação do consumo alimentar foi avaliada segundo os padrões específicos para pacientes com DRC (SOCIEDADE..., 2011). Os exames bioquímicos foram avaliados de acordo com as diretrizes clínicas para cuidado ao paciente com DRC (BRASIL, 2014), tais como: albumina (3,5 a $5,5 \mathrm{mg} / \mathrm{dL}$ ), fósforo (2,5 a 4,8 mg/dL), potássio (3,6 a $5,4 \mathrm{mEq} / \mathrm{L})$, cálcio $(4,25$ a $5,25 \mathrm{mg} / \mathrm{dL}$ ), ferritina (feminino: 11,0 a $306,8 \mathrm{ng} / \mathrm{mL}$ e masculino: 23,9 a $336,2 \mathrm{ng} / \mathrm{mL}$ ), sódio $(138,0$ a $146,0 \mathrm{mEq} / \mathrm{L})$, vitamina $D(>20,0 \mathrm{ng} / \mathrm{mL})$ e Kt/v $(\geq 1,20)$. As análises estatísticas foram feitas no programa estatístico Statistical Package for the Social Sciences for Windows (SPSS, versão 17.0). Na caracterização da amostra foi utilizada a análises descritiva, e na análise da comparação das médias foi usado o Test $t$ de Student bicaudal para as amostras independentes. Todos os testes foram realizados com o nível de significância de $5 \%$.

\section{RESULTADOS}

Participaram do estudo 122 idosos da região central do Estado do RS. Com maior distribuição de frequência, o sexo masculino, com idade $<80$ anos, cor branca, vivendo com companheiro, escolaridade < 5 anos, renda mensal $>2$ salários mínimos, com nefropatia diabética como principal doença de base e tempo de hemodiálise $\geq 3$ anos (Tabela 1).
Tabela 1 - Características sociodemográficas e hábitos de vida de idosos com DRC em hemodiálise da região central do Rio Grande do Sul. Santa Maria, RS, 2018 $(n=122)$

\begin{tabular}{|c|c|c|}
\hline Variáveis & $\mathbf{N}$ & $\%$ \\
\hline \multicolumn{3}{|l|}{ Sexo } \\
\hline Masculino & 72 & 59,0 \\
\hline Feminino & 50 & 41,0 \\
\hline \multicolumn{3}{|l|}{ Idade (anos) } \\
\hline$<80$ anos & 109 & 89,3 \\
\hline$\geq 80$ anos & 13 & 10,7 \\
\hline \multicolumn{3}{|l|}{ Cor da pele } \\
\hline Branca & 87 & 71,3 \\
\hline Negra & 24 & 19,7 \\
\hline Parda & 11 & 9,0 \\
\hline \multicolumn{3}{|l|}{ Estado conjugal } \\
\hline Com companheiro & 73 & 59,8 \\
\hline Sem companheiro & 49 & 40,2 \\
\hline \multicolumn{3}{|l|}{ Escolaridade (anos de estudo) } \\
\hline$<5$ anos & 83 & 68,0 \\
\hline$\geq 5$ anos & 39 & 32,0 \\
\hline \multicolumn{3}{|l|}{ Renda familiar } \\
\hline$\leq 2$ salários mínimos & 58 & 45,9 \\
\hline > 2 salários mínimos & 64 & 52,5 \\
\hline \multicolumn{3}{|l|}{ Doença renal de base } \\
\hline Nefropatia hipertensiva & 28 & 23,0 \\
\hline Nefropatia diabética & 44 & 36,0 \\
\hline Doença renal crônica não especificada & 41 & 33,6 \\
\hline Doença renal crônica em estágio final & 2 & 1,6 \\
\hline Rim policístico & 3 & 2,5 \\
\hline Transtorno renal túbulo intersticiais & 3 & 2,5 \\
\hline com rejeição de transplante & 1 & 0,8 \\
\hline \multicolumn{3}{|l|}{ Tempo de hemodiálise } \\
\hline$<3$ anos & 58 & 47,5 \\
\hline$\geq 3$ anos & 64 & 52,5 \\
\hline
\end{tabular}

Fonte: Elaborada pelas autoras.

Quanto às características antropométricas, verificou-se, na Tabela 2, maior frequência para peso, circunferência do braço e circunferência da panturrilha, todos adequados.

Quanto à Tabela 3, houve uma diferença estatisticamente significativa entre os parâmetros antropométricos e sexo somente para o IMC.

Já na Tabela 4 foi encontrada diferença estatisticamente significativa entre os parâmetros dietéticos e o sexo para as quilocalorias totais, proteína, carboidrato, fósforo, potássio.

Conforme observado na Tabela 5, há diferença estatisticamente significativa dos parâmetros bioquímicos segundo o sexo para vitamina $\mathrm{D}$ e Kt/v. 
Tabela 2 - Características antropométricas de idosos com DRC em hemodiálise da região central do Rio Grande do Sul. Santa Maria, RS, 2018 (n=122)

Variáveis

Índice de Massa Corporal - IMC $(\mathrm{Kg} / \mathrm{m} 2)$

Baixo peso

Peso adequado

Peso elevado

Circunferência do Braço - CB (cm)

Desnutrição

Eutrofia

Sobrepeso

Circunferência da Panturrilha - CP (cm)

Perda muscular

Adequado n $\%$

$39 \quad 32,0$

$48 \quad 39,3$

$35 \quad 28,7$

$35 \quad 28,7$

$56 \quad 45,9$

$31 \quad 25,4$

$28 \quad 23,0$

$91-74,6$

Fonte: Elaborada pelas autoras.

Tabela 3 - Média e desvio-padrão dos parâmetros antropométricos segundo o sexo de idosos em hemodiálise da região central do Rio Grande do Sul. Santa Maria, RS, 2018 (n=122)

\begin{tabular}{lccc}
\hline \multicolumn{1}{c}{ Variáveis } & \multicolumn{2}{c}{ Sexo } & \multirow{2}{*}{$\mathbf{P}$} \\
\cline { 2 - 3 } & Masculino $(\mathbf{n = 7 2})$ & Feminino $(\mathbf{n}=\mathbf{5 0})$ & \\
\hline Índice de massa corporal - IMC $\left(\mathrm{kg} / \mathrm{m}^{2}\right)$ & $26,69 \pm 4,22$ & $24,77 \pm 5,96$ & $0,039 *$ \\
Circunferência do braço - CB (cm) & $28,81 \pm 3,38$ & $28,24 \pm 5,06$ & 0,458 \\
Circunferência da panturrilha - CP $(\mathrm{cm})$ & $33,09 \pm 7,81$ & $31,75 \pm 3,87$ & 0,263 \\
\hline
\end{tabular}

$* p<0,05$.

Fonte: Elaborada pelas autoras.

Tabela 4 - Média e desvio-padrão dos parâmetros dietéticos segundo o sexo de idosos em hemodiálise da região central do Rio Grande do Sul ( $n=122)$

\begin{tabular}{|c|c|c|c|}
\hline \multirow{2}{*}{ Variáveis } & \multicolumn{2}{|c|}{ Sexo } & \multirow{2}{*}{$\mathbf{P}$} \\
\hline & Masculino $(n=72)$ & Feminino $(n=50)$ & \\
\hline Quilocalorias totais (Kcal) & $1665,17 \pm 546,28$ & $1310,27 \pm 567,92$ & $0,001 *$ \\
\hline Proteína (g) & $70,78 \pm 26,44$ & $52,44 \pm 24,30$ & $0,000^{*}$ \\
\hline Carboidrato (g) & $218,54 \pm 73,91$ & $181,29 \pm 81,54$ & $0,010^{*}$ \\
\hline Lipídeos (g) & $56,95 \pm 22,35$ & $56,51 \pm 96,24$ & 0,970 \\
\hline Fósforo (mg) & $897,19 \pm 323,40$ & $736,66 \pm 333,38$ & $0,009 *$ \\
\hline Potássio (mg) & $1885,70 \pm 744,24$ & $1576,52 \pm 769,55$ & $0,028^{*}$ \\
\hline Cálcio (mg) & $533,73 \pm 256,88$ & $506,65 \pm 277,02$ & 0,580 \\
\hline Ferro (mg) & $24,37 \pm 94,88$ & $7,60 \pm 3,99$ & 0,215 \\
\hline Sódio (mg) & $1632,24 \pm 778,09$ & $1414,90 \pm 899,59$ & 0,157 \\
\hline
\end{tabular}

Fonte: Elaborada pelas autoras.

Tabela 5 - Média e desvio-padrão dos parâmetros bioquímicos segundo o sexo de idosos em hemodiálise da região central do Rio Grande do Sul ( $n=122)$

\begin{tabular}{lccc}
\hline \multirow{2}{*}{ Exames Bioquímicos } & \multicolumn{2}{c}{ Sexo } & P \\
\cline { 2 - 3 } & Masculino $(\mathbf{n}=\mathbf{7 2})$ & Feminino $(\mathbf{n}=\mathbf{5 0})$ & 0,295 \\
\hline Albumina $(\mathrm{mg} / \mathrm{dL})$ & $3,78 \pm 0,33$ & $3,72 \pm 0,33$ & 0,146 \\
Fósforo $(\mathrm{mg} / \mathrm{dL})$ & $106,78 \pm 64,91$ & $127,39 \pm 90,89$ & 0,777 \\
Potássio $(\mathrm{mEq} / \mathrm{L})$ & $5,16 \pm 0,73$ & $5,11 \pm 0,89$ & 0,565 \\
Cálcio $(\mathrm{mg} / \mathrm{dL})$ & $4,67 \pm 0,79$ & $4,57 \pm 1,02$ & 0,077 \\
Ferritina $(\mathrm{ng} / \mathrm{mL})$ & $486,84 \pm 399,96$ & $883,39 \pm 1829,09$ & 0,224 \\
Sódio $(\mathrm{mEq} / \mathrm{L})$ & $139,74 \pm 3,37$ & $138,98 \pm 3,34$ & $0,028^{*}$ \\
Vit $\mathrm{D}(\mathrm{ng} / \mathrm{mL})$ & $31,56 \pm 11,32$ & $27,09 \pm 10,19$ & $0,000^{*}$ \\
Kt/v & $1,35 \pm 0,29$ & $1,63 \pm 0,31$ & \\
\hline${ }^{*} \mathrm{p}<0,05$. & & &
\end{tabular}

Fonte: Elaborada pelas autoras. 


\section{DISCUSSÃO}

O presente estudo investigou a relação entre os parâmetros nutricionais entre homens e mulheres idosos em tratamento hemodialítico. No parâmetro antropométrico foi encontrada relação do sexo com o IMC, diferente de um estudo realizado no Distrito Federal com pacientes de ambos os sexos e idade superior a 18 anos, que não observou diferenças na média ou na distribuição percentual dos grupos de IMC entre os sexos (BOUSQUET-SANTOS; COSTA; ANDRADE, 2019). Em um outro estudo, a população de ambos os sexos apresentou média com níveis adequados para o IMC, com $54 \%$ mostrando valores ideais para pacientes em HD (IMC > $\left.23 \mathrm{~kg} / \mathrm{m}^{2}\right)$, (FERRAZ et al., 2015).

O IMC é um dos métodos mais utilizados para a avaliação do estado nutricional, embora ele seja um parâmetro com limitações, uma vez que considera o peso pela estatura e não a composição corporal. Conforme o avanço da idade, ocorrem modificações fisiológicas do organismo: a perda muscular progressiva apresenta-se maior no sexo feminino do que no masculino, estimando-se uma redução de $5 \%$ até os 50 anos e $10 \%$ até os 80 anos de idade. No idoso há também diminuição das fibras musculares de contração rápida que tende a levar à fraqueza muscular e ocasionar quedas e fraturas (ESQUENAZI; SILVA; GUIMARÃES, 2014). Ocorre também um aumento na redistribuição da gordura corporal e maior acúmulo de gordura na região do tronco e vísceras e menor nos membros (SILVA; PEDRAZA; MENEZES, 2015).

No presente estudo, referente ao parâmetro dietético, foi encontrada relação do sexo com quilocalorias totais, proteínas, carboidratos, fósforo e potássio, diferente de um estudo em que não houve nenhuma diferença entre os sexos para os nutrientes avaliados, proteína, quilocalorias totais, cálcio, ferro, potássio e fósforo (LUZ et al., 2017). Os pacientes em tratamento hemodialítico são considerados um grupo de risco para a desnutrição energético-proteica (DEP), pois tendencionam a ter uma alimentação inadequada relacionada às recomendações nutricionais (ALVARENGA et al., 2016).

A causa da desnutrição é multifatorial, incluindo déficit alimentar, que se relaciona aos distúrbios hormonais e gastrointestinais, restrições dietéticas em razão do tratamento e uso de farmacoterapia, que alteram a absorção dos nutrientes (JUNIOR et al., 2019).

Segundo um outro estudo realizado em uma clínica de nefrologia em São Paulo, pacientes em tratamento hemodialítico possuem um inadequado consumo de energia, proteína, fósforo e potássio, e há diferença estatisticamente significante entre os sexos. Também se notou que os pacientes do sexo masculino tendem a consumir maior quantidade de nutrientes, como proteína, fósforo e potássio em relação às muIheres (MACHADO; BAZANELLI; SIMONY, 2014 ).

Quanto aos parâmetros bioquímicos, neste estudo foi encontrada relação do sexo com a vitamina $D$ e Kt/ $\mathrm{V}$, diferente de um estudo em que as variáveis determinadas, em relação aos sexos, tiveram diferenças para ferro sérico e creatinina (BARROS et al., 2019).

Quanto à vitamina $D$, um estudo de coorte retrospectivo de Canhos (2018) avaliou em pacientes em hemodiálise no Hospital das Clínicas de Botucatu (SP), no período de janeiro/2014 a janeiro/2017, a associação entre os níveis séricos de vitamina $D$ e mortalidade e identificou qual o valor de corte de vitamina D abaixo do qual ocorre aumento da mortalidade. Esses pacientes foram avaliados mensalmente por meio de exame clínico e laboratorial conforme protocolo da rotina do serviço de hemodiálise, sendo a dosagem sérica de vitamina $D$ realizada semestralmente, especificamente nos meses de janeiro (verão) e julho (inverno). Os resultados indicaram que no verão a média da vitamina $D$ foi de $27,6 \pm 11 \mathrm{ng} / \mathrm{mL}$ e no inverno de $25,5 \pm 8,95 \mathrm{ng} / \mathrm{mL}$. Foi encontrada associação entre o nível sérico de vitamina $D$ e mortalidade nos pacientes em hemodiálise, e a dosagem sérica menor ou igual a $23,6 \mathrm{ng} / \mathrm{mL}$ no verão associou-se à maior mortalidade por todas as causas. No presente estudo a média da vitamina $D$ foi superior ao valor de risco para mortalidade citado anteriormente, tanto para homens quanto para mulheres, porém os homens apresentaram resultados melhores que as mulheres.

Portadores de doença renal crônica possuem vários motivos para evoluir com baixas concentrações séricas de 25 -hidroxivitamina $D$. A baixa exposição solar, dieta restritiva e a própria evolução da doença renal, conduzem para a diminuição da vitamina D (WEN-CHIH et al., 2016). Consequentemente, esses fatores ocasionam questionamentos em relação aos efeitos prejudiciais dessa hipovitaminose, assim como aos efeitos benéficos da sua suplementação. Há divergência na literatura quanto aos benefícios da reposição de vitamina $D$ nos pacientes renais crônicos em hemodiálise, principalmente quanto ao seu efeito sobre a mortalidade (DAMASIEWICZ; TOUSSAINT, 2015). Além disso, não se conhece o valor de corte de 25-hidroxivitamina $D$ que se associa ao melhor prognóstico nesses pacientes (SOUBERBIELLE; CHAZOT, 2017).

Quanto ao Kt/V, nossos resultados assemeIham-se aos obtidos no Censo do Estado Nutricional de Pacientes em Hemodiálise, quando os pacientes, 
em sua maioria, estavam com peso adequado segundo o IMC e apresentavam valores de Kt/ $\mathrm{V}$ e albumina adequados (BOUSQUET-SANTOS; COSTA; ANDRADE, 2019).

Para adequação da diálise, é recomendado, pelas diretrizes clínicas para cuidado ao paciente com doença renal crônica, que se mantenha um valor de $\mathrm{Kt} / \mathrm{V}$ superior a 1,20. Na fórmula do Kt/V, o (K) representa a depuração de ureia do dialisador, que é multiplicada pelo tempo de tratamento $(t)$ e dividida pelo volume de distribuição de ureia do paciente (V) (BRASIL, 2014).

No presente estudo os resultados indicaram melhor adequação da diálise entre as mulheres do que nos homens, embora ambos estejam dentro dos valores recomendados. Resultados semelhantes foram encontrados na pesquisa de Bousquet-Santos, Costa, Andrade (2019).

Os achados deste estudo reforçam a importância da avaliação nutricional no contexto do tratamento de renais crônicos em hemodiálise, visando a uma boa adesão à dieta e adequação à diálise.

\section{CONCLUSÃO}

Os resultados do presente estudo revelam que os homens com doença renal crônica apresentam-se em maior número, e que a maioria dos participantes possui idade inferior a 80 anos e nefropatia diabética como doença de base.

Há diferença estatisticamente significativa entre homens e mulheres em relação aos seguintes parâmetros nutricionais: IMC, quilocalorias totais, proteína, carboidrato, fósforo e potássio, vitamina $\mathrm{D}$ e Kt/V.

Pode-se destacar que a pesquisa contribuiu para os estudos da nutrição em gerontologia, pois evidenciou a importância da avaliação do estado nutricional de idosos em hemodiálise, bem como da monitorização da adesão à dieta com acompanhamento pelos parâmetros bioquímicos.

O estudo apresenta algumas limitações que devem ser consideradas, especialmente pelo delineamento ser de um estudo transversal, não sendo possível relacionar a causalidade entre as variáveis.

\section{REFERÊNCIAS}

ALVARENGA, L. A. et al. Análise do perfil nutricional de pacientes renais crônicos em hemodiálise em relação ao tempo de tratamento. Jornal Brasileiro de Nefrologia, v. 39, n. 3, p. 283, 2016.
BARRETO, M. S.; CARREIRA, L.; MARCON, S. S. Envelhecimento populacional e doenças crônicas: Reflexões sobre os desafios para o Sistema de Saúde Pública. Revista Kairós: Gerontologia, v. 18, n. 1, p. 325-339, 2015.

BARROS, L. A. et al. Análise do perfil nutricional de pacientes submetidos à terapia hemodialítica em um município do leste maranhense. Revista Eletrônica Acervo Saúde, sup. 28, jul. 2019.

BORGES, N. D. S. et al. Envelhecimento e força muscular respiratória de idosos independentes residentes de uma instituição de longa permanência em regime aberto. Jornal de Ciências Biomédicas e Saúde, v. 1, n. 2, p. 61-67, 2015.

BOUSQUET-SANTOS, K.; COSTA, L. G.; ANDRADE, J. M. L. Estado nutricional de portadores de doença renal crônica em hemodiálise no Sistema Único de Saúde. Ciência \& Saúde Coletiva, v. 24, p. 1.189-1.199, 2019.

BRASIL. Ministério da Saúde. Diretrizes clínicas para o cuidado ao paciente com Doença Renal Crônica - DRC no Sistema Único de Saúde. Brasília: Ministério da Saúde, 2014. Disponível em: bvsms.saude.gov.br/bvs/publicacoes/diretrizes_clinicas_cuidado_paciente_renal.pdf. Acesso em: 15 ago. 2019.

CANHOS, M. M. S. Associação entre os níveis séricos de vitamina $D$ e mortalidade em hemodiálise: estudo de coorte. 2018. Dissertação (Dissertação em Fisiopatologia em Clínica Médica) - Universidade Estadual Paulista "Júlio de Mesquita Filho", Botucatu, 2018.

DA COSTA, T. H. M. CalcNut: plataforma para cálculo de dieta. 2009. Disponível em: https://fs.unb.br/nutricao/calcnut. Acesso em: 16 jul. 2019.

DAMASIEWICZ, M. J.; TOUSSAINT, N. D. Is nutritional vitamin D supplementation beneficial in dialysis patients? Clin J Am Soc Nephrol., v. 10, n. 4, p. 544-546, 2015.

DUARTE, I. M. R. L. Desnutrição no idoso. 2017. Tese (Doutorado) - Universidade de Coimbra, 2017.

ESQUENAZI, D.; SILVA, S. B.; GUIMARÃES, M. A. Aspectos fisiopatológicos do envelhecimento humano e quedas em idosos. Revista Hospital Universitário Pedro Ernesto, v. 13, n. 2, 2014.

FERRAZ, S. F. et al. Estado nutricional e ganho de peso interdialítico de pacientes com doença renal crônica em hemodiálise. Jornal Brasileiro de Nefrologia, v. 37, n. 3, p. 306-314, 2015.

JUNIOR, W. S. L. et al. Estado nutricional e níveis séricos de minerais em pacientes com insuficiência renal em hemodiálise (Nutritional Status and Serum Mineral Levels in Patients with Renal Insufficiency in Hemodiálise). Saúde em Foco, v. 6, n. 2, p. 40-51, 2019.

LUZ, C. A et al. Avaliação da ingestão nutricional de pacientes com doença renal crônica em tratamento hemodialítico. Braspen Journal, v. 32, n. 3, p. 241-245, 2017.

MACHADO, D. A.; BAZANELLI, P. A.; SIMONY, F. R. Avaliação do consumo alimentar de pacientes com doença renal crônica em hemodiálise. Revista Ciência \& Saúde, v. 7, n. 2, p. 76-84, 2014. 
MARINHO, F.; PASSOS, V. M. A.; FRANÇA, E. B. Novo século, novos desafios: mudança no perfil da carga de doença no Brasil de 1990 a 2010. Epidemiologia e Serviços de Saúde, v. 25, n. 4, p. 713-724, 2016.

MENDES, J. L. V. et al. O aumento da população idosa no Brasil e o envelhecimento nas últimas décadas: uma revisão da literatura. Remas - Revista Educação, Meio Ambiente e Saúde, v. 8, n. 1, p. 13-26, 2018.

MIRANDA, G. M. D.; MENDES, A, C, G; SILVA, A. L. A. O envelhecimento populacional brasileiro: desafios e consequências sociais atuais e futuras. Revista Brasileira de Geriatria e Gerontologia, v. 19, n. 3, p. 507-519, 2016.

NATIONAL KIDNEY FOUNDATION. About Chronic Kidney Disease. K/DOQI Clinical Practice Guidelines for Chronic Kidney Disease, 2019. Disponível em: https://www. kidney.org/atoz/content/about-chronic-kidney-disease. Acesso em: 13 jun. 2019.

OPAS. Organização Pan-Americana. REUNIÓN DEL COMITÊ ASESOR DE INVESTIGACIONES EN SALUD, 36., Encuestra Multicêntrica, Salud Beinestar y Envejecimeiento (Sabe) en América Latina y el Caribe. Informe preliminar. 2001. Disponível em: http://envejecimiento.csic.es/documentos/documentos/paho-salud-01.pdf. Acesso em: 3 maio 2020.

SESSO, R. C. et al. Brazilian chronic dialysis census 2014. Jornal Brasileiro de Nefrologia, v. 38, n. 1, p. 54-61, 2016. SILVA, N. A.; PEDRAZA, D. F.; MENEZES, T. N. Desempenho funcional e sua associação com variáveis antropométricas e de composição corporal em idosos. Ciência \& Saúde Coleti$v a$, v. 20, p. 3.723-3.732, 2015.

SOCIEDADE BRASILEIRA DE NUTRIÇÃO PARENTERAL E ENTERAL. Associação Brasileira de Nutrologia. Terapia nutricional para pacientes em hemodiálise crônica. Projeto Diretrizes. Associação Médica Brasileira e Conselho Federal de Medicina. Ago. 2011. Disponível em: https:// diretrizes.amb.org.br/_BibliotecaAntiga/terapia_nutricional_para_pacientes_em_hemodialise_cronica.pdf. Acesso em: 12 ago. 2019.

SOUBERBIELLE, J. G.; CHAZOT, C. Vitamin D in Chronic kidney disease and dialysis patients. Nutrients, v. 9, n. 4, p. 328-342, 2017.

THOMÉ, F. S. et al. Relatório do censo brasileiro de diálise crônica 2017. Jornal Brasileiro de Nefrologia, v. 41, n. 2, p. 208-214, 2019.

WEN-CHIH et al. Pleiotropic effects of vitamin D in chronic kidney disease. Clinica Chimica Acta, v. 453, p. 1-12, 2016.

WHO. World Health Organization. Physical status: the use and interpretation of anthropometry [text on the Internet]. Geneva: WHO, 1995. (Technical Report Series $\left.n^{\circ} 854\right)$. Disponível em: http://www.who.int/childgrowth/publications/ physical_status/en/. Acesso em: 16 jun. 2015. 